\title{
Participación presupuestaria: las experiencias de Albacete, Logroño y Madrid*
}

\author{
Carmen Pineda Nebot \\ Licenciada en Derecho y en Ciencia Política y de la Administración. \\ Consultora de Administraciones Públicas
}

Sumario: I. INTRODUCCIÓN. II. EXPERIENCIAS DE PARTICIPACIÓN PRESUPUESTARIA. III. VARIABLES DE DIFERENCIACIÓN. IV. CONCLUSIONES. V. BIBLIOGRAFÍA.

\section{INTRODUCCIÓN}

En los últimos años el Presupuesto Participativo se ha convertido en un tema de debate central en el ámbito de la política local, tanto en España como en otros países de la Unión Europea. Las razones para ello son variadas. Por una parte, el reconocimiento, a nivel internacional, de los modelos instaurados por el Partido de los Trabajadores brasileño en ciudades como Porto Alegre. El Orçamento Participativo es una de las manifestaciones más visibles de un intento por hacer una «nueva política», alejada de la tecnocracia, más social y con mayor participación de los ciudadanos. Por otra parte, el descenso generalizado, en el caso europeo, de los niveles de participación electoral, fenómeno achacable a la falta de interés de los ciudadanos por una forma de hacer política alejada de sus deseos. Todo ello hace que la participación directa de la ciudadanía en la gestión económica pueda suponer una recuperación de su interés por los asuntos públicos, al permitir este sistema no sólo una mayor implicación en la toma de decisiones, sino también al favorecer la legitimidad de la actuación (mayor respaldo social). Además, ofrece una vía de solución a las dificultades presupuestarias de los ayuntamientos, al identificar mejor las situaciones concretas, permitiendo el aumento de la eficacia y la eficiencia en términos de políticas de gestión.

Pero hay que tener en cuenta que la mayoría de las experiencias de participación presupuestaria puestas en marcha en Europa y en concreto en España, tienen poco que ver con la experiencia de Porto Alegre y con muchas experiencias similares de América Latina. El contexto social, económico y político es muy distinto aquí, y la dinámica de la democracia participativa también. Además, el fenómeno es muy heterogéneo. Los

\footnotetext{
* Este artículo es una versión revisada y actualizada de la ponencia del mismo titulo presentada en el VII Congreso Nacional de Ciencia Política celebrado en Madrid los días 21-23 de septiembre de 2005.
} 
países europeos tienen entre ellos bastantes diferencias en términos de cultura política, de competencia de los gobiernos locales, de forma presupuestal. Lo que se llama presupuesto participativo no es exactamente lo mismo en Francia, Italia, Alemania o España.

No hay una metodología que pueda llamarse «presupuesto participativo», ni tampoco unas metodologías que puedan ser consideradas como un conjunto de variantes alrededor de una base común como ocurre en las experiencias latino-americanas donde la metodología de Porto Alegre ha sido importada, adaptada y combinada con otras inspiraciones en centenares de ciudades. Incluso dentro de cada país, se pueden encontrar varias metodologías, aunque los intercambios y las discusiones tienden a favorecer cierta homogeneización. Por eso los presupuestos participativos no constituyen en Europa una metodología, sino más bien un tema político y un movimiento de institucionalización de la participación ciudadana alrededor del presupuesto público (Sintomer, 2004: 1).

Sin embargo la mayoría de las experiencias españolas, sobre todo las más antiguas, han utilizado la metodología de la experiencia de Porto Alegre y de otras ciudades latinoamericanas, aunque con ligeras variaciones y adaptaciones locales. Con el desarrollo de las experiencias y los intercambios crecientes, la tendencia en estos momentos no está en la homogenización, sino en el desarrollo de modelos mixtos que, según los objetivos y las culturas políticas, recurran a varias metodologías para intentar combinar las ventajas y corregir a los defectos de cada una. Sin embargo, el modelo de Porto Alegre sigue, y seguirá siendo probablemente la referencia central, porque todavía es la referencia política más conocida y más popular.

En este artículo comenzaré señalando los rasgos más distintivos de la experiencia de Porto Alegre y de las tres experiencias españolas elegidas, para pasar a referirme a las variables de diferenciación utilizadas para la comparación de las cuatro experiencias y el análisis de cada una de las experiencias a partir de éstas variables, para finalizar con unas pequeñas conclusiones.

\section{EXPERIENCIAS DE PARTICIPACIÓN PRESUPUESTARIA}

\section{a) Porto Alegre}

La metodología de Porto Alegre (1.300.000 habitantes) ha sido hasta ahora la más influyente, y previsible lo seguirá siendo en un futuro. $\mathrm{Y}$ 
aunque con el desarrollo de las experiencias y los intercambios crecientes, la tendencia no está en la homogenización, sino en el desarrollo de modelos mixtos que, según los objetivos y las culturas políticas, recurren a varias metodologías para intentar combinar las ventajas y corregir los defectos de cada una, la experiencia de Porto Alegre seguirá siendo probablemente la referencia central, porque conocida.

Sus características principales son conocidas (Sousa de, 2002; Gret/Sintomer 2003):

- Se basa sobre dos dimensiones, territorial (con las asambleas de barrio o de sector) y temática (con asambleas discutiendo de varias políticas públicas).

- Se fundamenta en la participación abierta a todos los ciudadanos, sin ningún status especial atribuido a las organizaciones comunitarias

- Articula el nivel micro local (barrio o aldea) y el de ciudad.

- Articula asambleas abiertas a todos los ciudadanos y consejos de delegados que constituyen la cumbre del proceso y lo monitorizan (combinación de democracia directa y representativa).

- Utiliza criterios formales de repartición de los recursos, dentro del marco de un reglamento explícito que puede ser modificado por los participantes (o sea, compatibilizando las decisiones y las reglas establecidas por los participantes con las exigencias técnicas y legales de la acción gubernamental, respetadas también las limitaciones financieras).

- Necesita un ciclo organizado de discusiones y toma de decisiones durante todo el año.

- Permite discutir principalmente sobre el presupuesto de inversión, y especialmente sobre las obras.

El cambio del equipo de gobierno de la ciudad, el PT perdió las últimas elecciones municipales en el 2004, puede provocar algunos cambios en la metodología de esta experiencia. La nueva administración ya ha anunciado un nuevo modelo de gestión pública: la Gobernanza Solidaria Local (GSL), que consiste en la creación, en cada una de las 16 regiones en las que se encuentra dividida la ciudad, de un espacio llamado Gobernanza Local. Según Cesar Busatto, Secretario de Coordinación Política y Gobernaza Local del Ayuntamiento de Porto Alegre, «la Gobernanza Local es 
un forum ejecutivo, no deliberativo; es una articulación en red que busca crear pactos de co-responsabilidad. En este espacio no hay debate, ni votación, ni delegados». Este espacio está formado por: los Consejos Municipales y los Forum Locales, Cámara de Vareadores, Tercer Sector, Universidades, Ministerio Público, Poder Judicial, Tribunal de Cuentas del Estado, Poder Ejecutivo del Estado y Federal, Comité Gestor del Gobierno Municipal, Iniciativa Privada y Presupuesto Participativo. Estos actores se repartirán la responsabilidad de concretar las demandas de la población con el poder público.

¿Cuál será entonces el papel del Presupuesto Participativo, que no es un actor y sí un espacio de deliberación y debate de la ciudad? Aunque no es posible saberlo todavía, sí que parece, que con estos cambios se puede producir una disminución del poder de deliberación de las Asambleas del Presupuesto Participativo, de los Forums de Delegados y de las reuniones del Consejo del Presupuesto Participativo.

De todas formas habrá que esperar un tiempo para ver como funciona este nuevo modelo de gestión y cómo reaccionan los vecinos si el nuevo equipo de gobierno pretende reducir sus espacios de deliberación y debate.

\section{b) Albacete ${ }^{1}$}

Albacete (153.636 hab.) $)^{2}$ comienza en el año 2000 a celebrar los Plenos de la Participación, la filosofía de los mismos es producto de la voluntad del nuevo equipo de gobierno ${ }^{3}$ de ir creando cauces de implicación de los ciudadanos en el desarrollo de la política municipal, para construir la ciudad entre todos. Este proceso iniciado, no está concluido y cada año se van incorporando nuevas aportaciones ${ }^{4} \mathrm{y}$, estudiando y modificando 5

\footnotetext{
${ }^{1}$ Quisiera agradecer la ayuda prestada por CARMEN CAMPOS (Ayuntamiento de Albacete), JAVIER AviLÉs (Coordinador Foro Participación de Albacete) y AnTONIO ManZANARES (miembro del Foro).

2 Todos los datos de población son del Padrón municipal de 2001.

3 Tras la pérdida por el PSOE de las elecciones municipales en 1995, la Agrupación Municipal del partido con su secretario general, MANUEl PÉREZ CASTELl, elaboraró un programa basado en la máxima cercanía posible con los vecinos, que se plasmó en lo que llamaron «Concejos Abiertos». Ellos fueron el origen de los Plenos de la Participación que posteriormente se convirtieron en Foros de Participación.

${ }^{4}$ Para supervisar el grado de ejecución de cada Pleno, a partir del II Pleno se creó la Comisión de Seguimiento que se reúne de forma periódica y que está formada por los representantes de los colectivos que intervienen en cada Pleno, miembros del Equipo de Gobierno municipal y técnicos del Ayuntamiento.
} 
aquellos procedimientos que no dan los resultados esperados. Los vecinos $^{6}$ pueden presentar propuestas ${ }^{7}$ sobre proyectos y servicios a través de una asamblea abierta a todos los ciudadanos. El consejo del Foro, integrado por representantes de asociaciones de diversos ámbitos (vecinos, cultura, educación, ecología, inmigrantes, etc.) y ciudadanos no organizados, después de estudiar las propuestas negocia con el ayuntamiento los proyectos que se realizarán, teniendo en cuenta los recursos del presupuesto y la viabilidad técnica y legal y, posteriormente hace un seguimiento de la ejecución de los proyectos aprobados. Para la clasificación de las propuestas, se han elaborado y aprobado este último año unos criterios (ver Anexo 1) de priorización ${ }^{8}$ que tienen en cuenta cuestiones como la necesidad de equipamientos urbanos, la población beneficiada, etc. El proceso de Albacete supera ya el simple debate sobre proyectos de inversión, al discutirse otros gastos e ingresos (tasas y precios públicos). El Foro ha asumido también un papel de mediador social entre la ciudadanía y el Gobierno Local (en el «conflicto del botellón», la subida del precio en las tarifas del agua...). Además han realizado un análisis sobre la infraestructura urbana que permite especificar las demandas de las asociaciones en el proceso.

Las dificultades encontradas para que converjan de una manera efectiva los tres ejes que sostienen el proceso9: el eje técnico, que comprende las áreas del ayuntamiento; el eje político y finalmente el Foro de la Par-

\footnotetext{
${ }^{5}$ El IV Pleno de la Participación, supuso un cambio en relación al III, al crearse el llamado Foro de la Participación. La misión de este foro era la elección en la asamblea de una mesa integrada por un representante de los distintos colectivos que habían participado en anteriores plenos de la participación, así como la elección de un coordinador, cuyo perfil debía ser el de una persona no identificada con ningún tipo de organización ni partido y con proyección ciudadana.

Posteriormente se eligió un consejo (compuesto por 27 personas) en el que están presentes representantes de los diferentes sectores agrupados por la temática de su trabajo habitual. A partir de la aprobación, por parte del Foro del Reglamento, del Foro de Participación pueden también formar parte del Consejo ciudadanos no organizados.

${ }^{6}$ Hasta la aprobación del Reglamento del Foro de Participación, el 17 de diciembre del 2003, sólo podían presentar propuestas las asociaciones, a partir de esa fecha lo pueden hacer también los ciudadanos no organizados.

${ }^{7}$ Al presupuesto de 2005 se presentaron en la Asamblea del Foro de la Participación 525 propuestas.

${ }^{8}$ Para el presupuesto del 2005 una comisión del consejo elaboro unos criterios para priorización de las propuestas, que se presentaron y aprobaron en el último Foro de la Participación.

${ }^{9}$ En el reglamento del Foro, se arbitró un canal para que estos tres ejes convergieran de una manera más efectiva, que fue lo que se denominó la oficina del presupuesto participativo, una mesa técnica. Pero esta mesa no se ha puesto todavía en marcha.
} 
ticipación, junto con la falta de encaje entre los tiempos de presentación de propuestas y los plazos que marca la ley para la elaboración de los presupuestos municipales, han llevado al Consejo del Foro de la Participación ha tomar la decisión de que para el presupuesto del 2006 no se presenten propuestas y se aproveche este año para adaptar el calendario del Foro $^{10}$ a los plazos del presupuesto municipal.

Para los presupuestos del año 2007, no sólo pretenden respetar los plazos de presentación de propuestas, sino también cuantificar las propuestas, para conocer mejor cuánto vale lo que se pide. Con ello el proceso ganará en credibilidad.

\section{c) Logroño ${ }^{11}$}

Logroño (145.099 habitantes) ha puesto en marcha en el 2005 los Presupuestos Ciudadanos, aprobando el día 2 de marzo, por acuerdo de la Junta de Gobierno Local, las normas ${ }^{12}$ para su aplicación.

Cualquier ciudadano, a través de sus colectivos o asociaciones o de forma individual, podrá presentar propuestas al Presupuesto Municipal del año $2006^{13}$. Estas propuestas ${ }^{14}$ se canalizan en la Oficina de Participación Ciudadana y posteriormente se remitirán a las respectivas Juntas de Distrito (las que tengan carácter territorial inferior al Distrito), al Consejo Social (las de ámbito superior al Distrito o las de ámbito de Ciudad) y a la Comisión Especial de Quejas y Sugerencias. Estos tres órganos, durante los meses de mayo y junio, valoran y priorizan las propuestas, posteriormente los técnicos municipales realizan una valoración de la via-

\footnotetext{
${ }^{10}$ A partir del 2006 las propuestas se recogerán de enero a mayo y en mayo-junio se presentara la propuesta de presupuesto participativo y se realizara la asamblea.

${ }^{11}$ Quisiera agradecer la ayuda prestada por ÁNGEL SÁINZ YANGÜELA (Teniente de Alcalde y Concejal de Participación del Ayuntamiento de Logroño).

${ }^{12}$ Con la aprobación de esta normativa interna, fruto del estudio comparativo de las experiencias de Presupuestos Participativos existentes en España, el Ayuntamiento de Logroño intenta dar un paso más en su cercanía al ciudadano.

${ }^{13}$ Se han presentado 3.334 propuestas por 1.554 ciudadanos individuales y 30 propuestas por colectivos. De las propuestas presentadas la mitad se refieren a temas de ciudad.

${ }^{14}$ Desde el 15 de marzo al 15 de mayo los ciudadanos podían entregar sus propuestas a través de los distintos canales de participación ciudadana: Oficina de Participación Ciudadana, servicio 010, la web municipal y los buzones de sugerencias instalados en las dependencias municipales. También se podían entregar directamente a los concejales, a los miembros de la Juntas de Distrito o a los colectivos y asociaciones de la ciudad.
} 
bilidad técnica, jurídica y económica de cuyos resultados informarán a las Juntas de Distrito para que puedan realizar una nueva priorización, si así lo estiman. Una vez evaluadas todas las propuestas recibidas, la Junta de Gobierno Local las estimará en función de criterios objetivos como el interés general, urgencia, población a la que afecta... A continuación se incorporarán las propuestas al presupuesto municipal, que será valorado socialmente por el Consejo de la Ciudad y finalmente, se aprobará en Pleno.

Una vez terminado el proceso y antes de comenzar con la elaboración del siguiente presupuesto habrá una fase de información y análisis donde además de divulgar el alcance del presupuesto ciudadano y de difundir el contenido del presupuesto municipal se hará la valoración de la implicación de los ciudadanos en la experiencia y se elaboraran unas conclusiones sobre el primer año de la experiencia.

En lo que respecta a la parte del presupuesto municipal en el que los ciudadanos están participando, señalar que el art. 58 del Reglamento Orgánico de Participación Ciudadana del Ayuntamiento de Logroño habla del 5\% de los gastos previstos para inversión en el Presupuesto Municipal, como cifra que gestionarán el conjunto de las Juntas de Distrito ${ }^{15}$. De esa cantidad es de la que extraen la cuantía, pero eso no quiere decir que queda afectada a un gasto del capítulo VI (inversión). Por otra parte, ese 5\% es exclusivamente para las Juntas de Distrito, de manera que el Consejo Social puede admitir alguna propuesta que no se descontará de esa cantidad ${ }^{16}$.

Es una experiencia muy reciente por lo que habrá que esperar unos años para valorar sus resultados. Lo que diferencia a esta experiencia es la utilización de la Ley 57/2003, de 16 de diciembre, de Medidas para la Modernización del Gobierno Local ${ }^{17}$, para poner en marcha la participa-

15 El capitulo VI de gastos del Presupuesto Municipal de 2005 del Ayuntamiento de Logroño es de 55.882.000 euros, por lo tanto la cantidad que gestionaran las Juntas de Distrito será de 2.794.110 euros.

16 La cantidad que se tenía prevista era de 3 millones de euros pero al finalizar el proceso y ante las numerosas propuestas presentadas la cantidad ha aumentado hasta 7 millones.

17 La Ley 57/2003, de 16 de diciembre, de Medidas para la Modernización del Gobierno Local obliga en su artículo 128 a los ayuntamientos de un determinado tamaño a «crear distritos como divisiones territoriales propias, dotadas de órganos de gestión desconcentrada, para impulsar y desarrollar la participación ciudadana en la gestión de los asuntos municipales y su mejora....» Correspondiéndole al Pleno de la Corporación, la creación de los distritos y su regulación, así como determinar...»el porcentaje mínimo de los recursos presupuestarios de la corporación que deberán gestionarse por los distritos, en su conjunto». 
ción de los ciudadanos en el proceso presupuestario. Con esta ley se ha creado un marco legal para la participación territorial de los ciudadanos en el presupuesto, inexistente hasta ahora en la legislación española (Pineda, 2004: 3) que el gobierno de la ciudad de Logroño ha utilizado y que suponemos que será también utilizado por otros municipios.

\section{d) Madrid ${ }^{18}$}

La llegada al Ayuntamiento de Madrid (2.9387.723 habitantes) de un nuevo equipo de gobierno ${ }^{19}$, en las últimas elecciones municipales, ha cambiado sustancialmente los mecanismos de gestión. Un ejemplo de ello son los Planes Especiales de Inversión y Actuación Territorial 2004-2008, estos planes cuyo objetivo prioritario es el reequilibrio social y territorial de la ciudad han sido elaborados de forma conjunta y consensuada con los representantes de los vecinos a los que directamente iban dirigidos.

Los Planes Especiales de Inversión y Actuación Territorial ${ }^{20}$ son programas de intervención territorial que desarrollan diversas actuaciones ${ }^{21}$ en aquellos distritos ${ }^{22}$ que precisan de actuaciones preferentes y lo hacen con la participación de los ciudadanos en su diseño y gestión. Los distritos seleccionados ${ }^{23}$ han sido: Carabanchel, San Blas, Tetuán y Vicálva$\mathrm{ro}^{24}$.

La elaboración se ha llevado a cabo a través del trabajo conjunto del Ayuntamiento de Madrid ${ }^{25}$, representado por la Concejalía de Economía y

${ }^{18}$ Quisiera agradecer la ayuda prestada por Victor García Segador (Director General de Participación Ciudadana del Ayuntamiento de Madrid)

19 Aunque volvió a ganar por mayoría absoluta el Partido Popular, el perfil del nuevo Alcalde, AlBerto RuIZ- Gallardón, ha provocado importantes cambios políticos y de gestión.

${ }^{20}$ No se han definido unas cifras, sino que las cantidades han venido dadas por la suma de las actuaciones a realizar.

${ }^{21}$ Actuaciones que requieren recursos extraordinarios.

${ }^{22}$ Los distritos han sido seleccionados conjuntamente por el Ayuntamiento y la Federación Regional de Asociaciones de Vecinos de Madrid.

${ }^{23}$ Estos cuatro distritos suman una población de 594.396 habitantes, lo que representa el $20 \%$ de la población madrileña.

${ }^{24}$ Con posterioridad se ha incorporado el distrito de Usera. Se piensa en incorporar más adelante otro distrito y barrios de algunos distritos.

${ }^{25}$ Durante el año 2004 el Ayuntamiento de Madrid asignó 3 millones de euros para la realización de los estudios y proyectos que permitieran la iniciación de este proyecto. 
Participación Ciudadana y las Juntas de Distrito, y la Federación Regional de Asociaciones de Vecinos, en cuatro fases: organización y lanzamiento, diagnóstico, propuestas y programación y aprobación.

La metodología utilizada se ha basado en tres principios:

- Diagnóstico participativo de la situación ${ }^{26}$ y necesidades de los distritos

- Incorporación en todo el proceso del movimiento ciudadano

- Toma de decisiones a través del trabajo conjunto y el consenso

En la fase de ejecución se han constituido dos comisiones, una de Coordinación Administrativa que tiene como objeto la coordinación de todas las áreas administrativas involucradas en los Planes, y una de Seguimiento, formada por la administración y representantes del movimiento vecinal y ciudadano, que tiene funciones de información, seguimiento, sugerencias y evaluación del proceso y de los resultados. Con ello se pretende que la participación sea continua en todo el proceso.

La experiencia no ha hecho más que comenzar y como se sabe es necesario darle tiempo para conocer sus resultados y también para ver si va a ser una actuación puntual o se va a continuar en esa dirección.

\section{VARIABLES DE DIFERENCIACIÓN}

$\mathrm{Al}$ estudiar las experiencias de participación presupuestaria existentes tanto en España como en otros países vemos que presentan diferencias entre ellas. Para facilitar la comprensión de estas diferencias, he elegido dos variables de diferenciación ${ }^{27}$ : objetivos y actores. En primer lugar plantearé las variables que voy a utilizar para luego observarlas en las experiencias a comparar.

\footnotetext{
${ }^{26}$ En la fase de recogida de información para el diagnóstico se llevaron a cabo en cada uno de los distritos reuniones con el movimiento sectorial. Estas reuniones fueron convocadas por las diferentes Juntas de Distrito y con los criterios propios de cada responsable en lo que respecta a su composición y calendario.

${ }^{27}$ Para comparar las experiencias existen muchas variables de diferenciación, pero en este caso se utilizan las de la Investigación Internacional Comparada sobre Democracia Participativa que se está realizando en el Centro MARC BLOCH de Berlín, coordinado por el profesor YveS Sintomer, con el apoyo de la Fundación Hans- Böckler y la Delegación de la Ciudad del gobierno francés.
} 


\section{a) Objetivos}

Los objetivos son muy distintos pero se puede diferenciar a nivel analítico tres tipos potenciales que resumen los varios objetivos concretos que explicitan los actores (Gret/Sintomer, 2003; Bacqué/Rey/Sintomer, 2004):

- Nivel administrativo, se puede utilizar la participación para mejorar la gestión pública.

- Nivel social, se puede pensar que la participación presupuestaria va a influir sobre las relaciones sociales.

- Nivel político, parece posible convertir esta metodología innovadora en un instrumento para «democratizar la democracia», como dicen los brasileños de Porto Alegre.

\section{Nivel administrativo}

Este nivel es fundamental en Brasil. Probablemente porque se pueden constatar efectos concretos de los presupuestos participativos en términos de lucha contra la corrupción, de reducción del clientelismo y de modernización de la gestión pública.

Pero la relación no es automática; en los países europeos más interesados en modernizar su Estado, como los de Europa del Norte o el Reino Unido, el desarrollo del presupuesto participativo es casi inexistente. Aún más, en el interior de un mismo país las ciudades más «modernas» a nivel administrativo no son siempre las que tienen un presupuesto participativo, y viceversa, como se ve muy bien en España.

Sin embargo, los análisis (Sintomer, 2004) realizados demuestran que la introducción de una experiencia de participación presupuestaria está casi siempre relacionado con un proceso de modernización administrativa, sea porque para éste se necesita el uso de un instrumento fuerte de participación, sea porque el desarrollo de la participación presupuestaria no puede seguir adelante sin trasformar la administración.

Esta transformación puede realizarse de distintos modos. Uno de ellos es el de mejorar la gestión de proximidad, articulando la cooperación trasversal de los distintos servicios públicos en un territorio, permitiendo un acceso más fácil a estos servicios, nombrando en cada distrito un responsable administrativo y un responsable político para seguir todo los problemas y solicitudes de la ciudadanía, y desarrollando la participación para conocer mejor las necesidades de la gente. Otro es más ambicioso, pues lo que pre- 
tende es un proceso de modernización general y la participación se concibe como una de las vías de la modernización administrativa; La idea es introducir más trasversalidad en las administraciones, ofrecer servicios más accesibles, tener más en cuenta las demandas de la gente, proponer objetivos de calidad, desarrollar un presupuesto por objetivos en vez del presupuesto clásico, y reformar el management interno de la administración.

\section{Nivel social}

En Brasil el presupuesto participativo es un instrumento de las clases populares, un método privilegiado para permitir una inversión de las prioridades a favor de los pobres y también de las mujeres. No ocurre lo mismo en España, aunque eso no significa que no haya objetivos sociales, pero éstos son distintos. Existen en las experiencias españolas temas de inclusión social y de carencias de servicios e infraestructuras pero no hay mecanismos para la movilización de los más desfavorecidos. Tampoco se ha estudiado hasta ahora si la introducción de la participación presupuestaria puede realmente modificar las prioridades sociales, como ha ocurrido en el caso en Porto Alegre (Marquetti, 2001).

\section{Nivel político}

La reflexión sobre la democracia constituye uno de los puntos principales de la agenda política en el inicio del siglo XXI. Si el siglo anterior estuvo marcado por la afirmación democrática como elemento central de la política, el debate contemporáneo da un paso más y se preocupa de la calidad de estos regímenes.

La necesidad de reflexionar sobre la calidad de las democracias contemporáneas se relaciona con la crisis del Estado, especialmente las crisis vividas por sus instituciones representativas. El debate sobre la Reforma del Estado debe abarcar una amplia discusión sobre los instrumentos por medio de los cuáles la democracia debe discurrir. Se trata de la necesidad de buscar mayor legitimidad en los procesos democráticos, fomentando, con eso, gestiones públicas transparentes y mayor responsabilidad.

Entre los problemas más evidentes está la distancia entre representante y representado, el no cumplimiento de las promesas de campaña y la ausencia de mecanismos de control de los representantes por parte de los representados. El reconocimiento de esas dificultades y el debate sobre sus soluciones llevan a que se replanten la naturaleza y las estructuras de la representación política. Entre las formas alternativas que se plantean 
están las experiencias de democracia semidirecta o participativa, la democracia representativa puede combinarse con democracia participativa que facilita mayor presencia de los ciudadanos en el proceso de toma de decisiones. Los dos modos de ejercicio de poder no constituyen sistemas excluyentes, sino complementarios y compatibles ${ }^{28}$.

Esta complementariedad no significa, sin embargo, ausencia de conflictos. El fortalecimiento de mecanismos de participación directa o semidirecta lleva a una nueva configuración de poder y pone en evidencia las deficiencias del sistema representativo. La apertura de espacios de participación ciudadana provoca una tensión constante entre las dos legitimidades (la de los representantes y la de los representados) y es necesario encontrar un punto de equilibrio entre ambas.

Para los responsables de Porto Alegre el presupuesto participativo es un buen ejemplo a escala local de lo que sería, en realidad, un nuevo modelo de democracia; es decir una nueva forma de relacionarse el Estado con los ciudadanos y con los distintos agentes existentes en la sociedad. Se trataría de uno de los elementos, tal vez el principal, de una respuesta a la crisis del Estado en su forma generada por la modernidad (Genro, 2000) Para ello se ha creado un sistema que completa la democracia representativa con elementos de democracia directa. Lo que implica la emergencia de un cuarto poder, el de los ciudadanos cuando toman las decisiones directamente o a través de delegados estrechamente controlados; un poder que no sustituye sino que se articula con los tres poderes clásicos: el ejecutivo, el legislativo y el judicial (Bacqué/Rey/Sintomer, 2004).

En cambio, en Europa, la mayoría de las experiencias de presupuesto participativo no son todavía deliberativas: son consultivas y las decisiones las toman al final los políticos. Ocurre siempre así en Francia, Alemania, Bélgica, Inglaterra y Portugal, por los menos cuando se trata de decisiones más allá del nivel de barrio. En España, la situación es intermedia entre la de los otros países europeos y la de Porto Alegre.

\section{b) Actores}

Dos diferencias importantes separan los presupuestos participativos españoles del de Porto Alegre ${ }^{29}$. La primera es que, en España, son casi

\footnotetext{
${ }^{28}$ Recordemos que la Carta Europea de Autonomía Local garantiza expresamente la democracia directa y defiende su coexistencia con la democracia representativa.

${ }^{29}$ En muchas ciudades brasileñas y latino-americanas, los presupuestos participativos vienen también desde arriba. Cf. Avritzer, 2004, y AvritZer/Navarro, 2003.
} 
siempre procesos «desde arriba». La confluencia entre un movimiento desde abajo y uno desde arriba, que se dio en Porto Alegre, no suele darse, con la excepción del caso de Albacete.

La segunda diferencia es que, en Porto Alegre y en casi todas las experiencias latino-americanas, son las clases populares los actores principales del proceso - este hecho provoca las criticas de una parte de las clases altas a quienes no les gusta este «instrumento de los pobres». En cambio, en España, son las clases medias las que son las más activas, siendo los jóvenes, los extranjeros o inmigrantes y los más pobres los que se quedan ausentes o marginados en los presupuestos participativos.

Si miramos a los actores más concretos, hay que destacar en España el papel fundamental de los partidos políticos. De hecho, son más bien dirigentes locales de los partidos los que están realmente activos, porque no existe ningún país en que un partido haya aplicado experiencias de participación presupuestaria en todos los municipios en los cuales detenta el poder. Se piensa que son los partidos de izquierda los que están más interesados en la temática, aunque de los tres municipios que hemos estudiado sólo uno de ellos, Albacete, esta gobernado por un partido de izquierdas.

Las fundaciones y las $\mathrm{ONGs}^{30}$ juegan también, en muchos países, un papel importante en la difusión de la idea de presupuesto participativo, en la elaboración de las metodologías necesarias y en el asesoramiento técnico del proceso. En España, excepto en Cataluña (Fundación Jaime Bofill...), no han participado de ninguna manera.

Un tercer tipo importante de actores son las asociaciones y los varios colectivos de vecinos y de ciudadanos. Muchas metodologías están concebidas para implicar a los ciudadanos que no están organizados y no son muy favorables a los colectivos. En muchas partes, esto ha generado tensiones entre el presupuesto participativo y las asociaciones, hasta provocar una casi parálisis del proceso, como ha ocurrido en Córdoba. En otros lugares, donde la metodología es más favorable a la participación de las asociaciones, éstas juegan un papel más dinámico, y a veces central, como en Albacete donde ocupan casi todo el espacio. En el caso de Madrid, el papel central lo ocupa un tipo de asociaciones: las de vecinos.

\footnotetext{
${ }^{30}$ Las Fundaciones han sido tal vez el actor principal en Alemania a nivel nacional, y una pequeña ONG llamada «Arbeitsgruppe Burgerhaushalt Berlín» ha sido muy activa en la capital del país. En Francia, dos ONGs nacionales, l'ADELS (que ofrece cursos de capacitación en temas de gestión local y publica la revista Territoires) y DRD (Democratizar Radicalmente la Democracia) han hecho un trabajo importante. En Inglaterra, la asociación «Community Pride Initiative» ha sido el actor decisivo en el proceso de Salford.
} 
Asimismo hay que referirse a que la implicación de los funcionarios y técnicos no es homogénea. Las personas que se ocupan directamente del presupuesto participativo o que están encargadas de las relaciones con la ciudadanía juegan un papel muy activo y, de hecho, son las que hacen que el mecanismo pueda funcionar. Pero todavía, la gran mayoría de los funcionarios tienen dificultades para incorporar la participación en su trabajo cotidiano: porque tienen miedo a perder su poder, porque piensan que la gente podría amenazar la racionalidad administrativa o porque, simplemente, no han sido formados para esta problemática.

La última diferencia notable entre las experiencias de participación presupuestaria española y la de Porto Alegre (y de los demás casos latinoamericanos) es el papel del consejo municipal. En América Latina, el legislativo local se queda marginado en el proceso del presupuesto participativo, que se basa sobre todo en un diálogo entre el ejecutivo local y la sociedad civil. El sistema presidencialista que se aplica en casi todas las ciudades latino-americanas, con la elección directa del alcalde, contribuye probablemente a esta situación. En cambio, en España, donde el sistema político local es parlamentarista, en el cual el alcalde es elegido por el pleno municipal y comparte entonces el mismo color político, el pleno municipal juega normalmente un papel mayor que en América Latina. Por supuesto, el impulso del alcalde (o de los tenientes alcaldes) es fundamental, y muchas veces es él quien toma las decisiones reales.

En la tabla siguiente podemos las diferencias existentes entre las cuatro experiencias: la de Porto Alegre (Brasil) y tres españolas (Albacete, Logroño y Madrid).

En primer lugar, en lo que respecta al tamaño, vemos que aunque Madrid es la de mayor tamaño su experiencia sólo se aplica a una parte de su territorio, cuatro distritos, que representan el $20 \%$ de su población total. Por tanto es la experiencia de Porto Alegre la que se aplica a un municipio de mayor tamaño. Albacete y Logroño tienen un tamaño muy similar.

Aunque existen varios objetivos en todas las experiencias, lo cierto es que en cada una de ellas hay un objetivo que prima sobre los demás, aunque en el caso español por el poco tiempo transcurrido desde la puesta en marcha de las experiencias puede ir modificándose. De las tres experiencias españolas estudiadas es la de Albacete la que tiene en este punto más proximidad con la experiencia de Porto Alegre. Las otras dos se enmarcan más dentro de un proceso de modernización de la gestión local, en la que se encuentran inmersos muchos municipios españoles. 


\begin{tabular}{|c|c|c|c|c|}
\hline & Porto Alegre & Albacete & Logroño & Madrid \\
\hline Población & 1.300 .000 & 153.636 & 145.099 & $\begin{array}{l}2.9387 .723 \\
594.396^{*}\end{array}$ \\
\hline $\begin{array}{l}\text { Objetivo } \\
\text { Principal }\end{array}$ & $\begin{array}{l}\text { Político } \\
\text { Social }\end{array}$ & $\begin{array}{l}\text { Político } \\
\text { Social }\end{array}$ & $\begin{array}{l}\text { Modernización } \\
\text { Descentralización }\end{array}$ & $\begin{array}{l}\text { Mejora de la } \\
\text { gestión pública } \\
\text { Social }\end{array}$ \\
\hline $\begin{array}{l}\text { Actores } \\
\text { impulsores del } \\
\text { proceso }\end{array}$ & $\begin{array}{l}\text { Confluencia entre un } \\
\text { movimiento desde } \\
\text { abajo y uno desde } \\
\text { arriba }\end{array}$ & $\begin{array}{l}\text { Confluencia entre } \\
\text { un movimiento } \\
\text { desde abajo y uno } \\
\text { desde arriba }\end{array}$ & Desde arriba & Desde arriba \\
\hline $\begin{array}{l}\text { Proporción del } \\
\text { presupuesto }\end{array}$ & $\begin{array}{l}\text { Fundamentalmente } \\
\text { sobre inversiones, } \\
\text { aunque se discute } \\
\text { sobre todo el gasto }\end{array}$ & $\begin{array}{l}\text { Todo el } \\
\text { presupuesto de } \\
\text { gasto, pero sin } \\
\text { mucha } \\
\text { concreción. } \\
\text { También las tasas } \\
\text { y los precios } \\
\text { públicos. }\end{array}$ & $\begin{array}{l}\text { El 5\% del gasto en } \\
\text { inversión del } \\
\text { presupuesto } \\
\text { municipal. }\end{array}$ & $\begin{array}{l}\text { Cantidad } \\
\text { necesaria para las } \\
\text { actuaciones } \\
\text { consensuadas }\end{array}$ \\
\hline $\begin{array}{l}\text { Ámbito de } \\
\text { aplicación }\end{array}$ & $\begin{array}{l}\text { Zona, temática y } \\
\text { ciudad }\end{array}$ & Ciudad & Distrito y ciudad & Algunos distritos \\
\hline $\begin{array}{l}\text { Actores } \\
\text { principales }\end{array}$ & $\begin{array}{l}\text { Ciudadanos } \\
\text { individuales }\end{array}$ & Asociaciones & $\begin{array}{l}\text { Ciudadanos } \\
\text { individuales }\end{array}$ & $\begin{array}{l}\text { Asociaciones de } \\
\text { vecinos }\end{array}$ \\
\hline $\begin{array}{l}\text { Partido Político } \\
\text { que impulsa la } \\
\text { experiencia }\end{array}$ & $\begin{array}{l}\text { Partido de los } \\
\text { Trabajadores }\end{array}$ & $\begin{array}{l}\text { Partido Socialista } \\
\text { Obrero Español }\end{array}$ & Partido Popular & Partido Popular \\
\hline $\begin{array}{l}\text { Fundaciones y } \\
\text { ONGs }\end{array}$ & Sí & No & No & No \\
\hline $\begin{array}{l}\text { Estructura } \\
\text { administrativa } \\
\text { especial }\end{array}$ & Sí & No & No & No \\
\hline
\end{tabular}

Fuente: Elaboración propia.

* La experiencia sólo implica a la población de cuatro distritos.

La similitud entre Porto Alegre y Albacete se mantiene también en el siguiente apartado, ya que a diferencia de lo que ocurre con casi todas las experiencias españolas e incluso europeas, en las que el proceso surge siempre desde arriba, o sea la iniciativa la toma el alcalde y su equipo y, muchas veces tiene problemas para movilizar a los ciudadanos que tienen dudas sobre los efectos reales de la participación, en el caso de Albacete la experiencia surgió de la confluencia de un movimiento desde arriba con uno desde abajo.

Quizá sobre esta cuestión habría que hacer alguna matización, todas las experiencias surgen porque el alcalde y en algunos casos su equipo de gobierno creen que la participación de los ciudadanos es positiva para el desarrollo de la ciudad. Si los representantes políticos no son capaces de compartir alguna parcela de su poder con los ciudadanos no es posible llevar a cabo una experiencia de este tipo. Pero en algunos casos se produce porque hay un encuentro entre las ideas participativas del responsable político y de los movimientos sociales de la ciudad, este es el caso de Albacete. 
En cuanto a la parte del presupuesto que es discutida o priorizada por los ciudadanos no existe un modelo común, sino que tiene que ver con las características propias de cada experiencia. El análisis de las experiencias más antiguas demuestran que con el tiempo cada vez se amplia más el campo de discusión, al estar en casi todos los casos los gastos en relación unos con otros (ejem.: un gasto en inversión provoca un gasto corriente o un gasto en personal...) Lo que está claro es que cuanto más elevada sea la parte del presupuesto (en valores absolutos y en porcentaje del total)sometida a discusión, ello es mejor para la ciudadanía. Sin embargo, no existen evidencias de una correlación directa entre el nivel de recursos debatidos y el nivel de participación, en términos cualitativos y cuantitativos. Tener pocos recursos para someter a discusión no es un obstáculo definitivo o un condicionante. Lo que cuenta es hacer transparente el monto, por muy reducido que éste sea, explicar por qué es limitado y que la población defina reglas claras de juego para su asignación.

También existen diferencias en el ámbito de aplicación. Porto Alegre comenzó su experiencia territorialmente (dividiendo la ciudad en dieciséis zonas o distritos) ampliando con el tiempo la participación a áreas temáticas y por último el conjunto de la ciudad. Por el contrario Albacete ha seguido el camino contrario, primero han sido los foros de ciudad, más adelante los foros temáticos (aunque sin regularidad) y ahora se están planteando los foros territoriales (de distrito o barrio). En el caso de Logroño se da un sistema mixto, territorial (distritos) y de ciudad, aunque con un predominio claro del territorial. Madrid es muy distinto a los demás, pues es una experiencia que no se da en todo el territorio del municipio sino sólo en una parte de él que se encuentra en una situación de desigualdad.

Los actores principales del proceso en Porto Alegre y Logroño son los ciudadanos individuales aunque también tienen protagonismo las asociaciones, en cambio en Albacete son las asociaciones las que asumen un mayor protagonismo y en Madrid es la Federación Regional de Asociaciones de Vecinos.

Aunque se piensa que son los partidos de izquierda los que están más interesados en la temática, de las tres experiencias que hemos estudiado sólo una de ellas, la de Albacete, esta gobernado por un partido de izquierdas.

En España las fundaciones y las ONGs no juegan, como ha ocurrido en Porto Alegre y en otros países europeos, un papel importante en la difusión de la idea de presupuesto participativo, en la elaboración de las meto- 
dologías necesarias y en el asesoramiento técnico del proceso. Ello debería ser un motivo de reflexión.

Por último, señalar que en general los municipios españoles no están todavía suficientemente preparados organizativa y técnicamente para llevar a cabo con éxito las experiencias de participación presupuestaria, aunque poco a poco van transformándose movidos por el desarrollo de la participación.

\section{CONCLUSIONES}

Es importante señalar, como hemos podido ver de las experiencias estudiadas, que no existe una receta universal para iniciar una experiencia de participación presupuestaria. Ésta tiene que adecuarse a la situación y a las características locales.

Pero sí parece recomendable hacer primero un diagnóstico de situación para ver hasta qué punto los principios rectores (Participación, Transparencia, Igualdad, Tolerancia, Eficacia y eficiencia, Equidad, Competitividad y Respeto a los acuerdos) son respetados y las pre-condiciones reunidas. Este diagnóstico va a variar de una ciudad a otra.

Un segundo momento importante es hacer, también en forma participativa, un mapa (o evaluación) de los actores locales interesados y de los actores opuestos al proceso.

Un tercer momento es el análisis y definición clara por parte del equipo de gobierno, del volumen y origen de los recursos que serán puestos a disposición de la experiencia y los que serán necesarios para que el municipio implante el proceso. Para implementar una experiencia de participación presupuestaria en buenas condiciones se necesitan cuatro tipos de recursos: a) un equipo municipal comprometido y capacitado para conducir el proceso b) recursos para comunicación e información amplia a la población y, c) un grupo técnico municipal para los estudios de viabilidad técnica, económica y presupuestaria de las demandas priorizadas. Las ciudades que no han pensado en estos recursos, por lo general, se han enfrentado a dificultades y en varios casos incluso han tenido que interrumpir el proceso. En ese momento es recomendable que los municipios realicen un estudio costo/beneficio o costo/resultados esperados.

Un cuarto momento es la construcción de alianzas y la ampliación de los diálogos para que la idea gane más adeptos y legitimidad en el municipio. Los elementos claves pueden ser: 
- Construir un acuerdo en el interior del gobierno

- Dialogar con los actores más relevantes de la sociedad civil

Un quinto momento será la elaboración de un reglamento interno que defina las reglas de juego, para el primer año

Es importante también tener en cuenta los principios operativos siguientes:

- Participación universal: Todos los ciudadanos/as deben poder participar, independientemente de su condición socioeconómica. Sin embargo, los ciudadanos organizados deben jugar un papel importante en el proceso. Además, es necesario pensar en medios específicos para involucrar a los ciudadanos más marginados.

- Transparencia del presupuesto, tanto de los ingresos como de los gastos.

- Flexibilidad: el proceso debe ser flexible para evaluarse y ajustarse continuamente.

- Objetividad: se debe usar criterios objetivos y explícitos para la asignación de recursos a fin de garantizar la credibilidad del proceso.

- Discriminación positiva y acciones afirmativas para asegurar la participación y el poder de decisión de los excluidos, (como por ejemplo los emigrantes).

Seguir todos estos pasos no supone la ausencia de dificultades, aunque si el que éstas se amortigüen. Algunas de las principales dificultades con las que se encuentran aquellos municipios que ponen en marcha una experiencia de este tipo son: la incapacidad del aparato administrativo para implantar el proceso, en la mayoría de los casos, las administraciones no están adaptadas a las nuevas exigencias impuestas: otro tipo de diálogo con la ciudadanía, trabajar fuera de los horarios convencionales, el trabajo en los barrios, etc. ; los sistemas de información a la ciudadanía y la baja calidad de la información que se presenta a los participantes; a la acumulación de obras aprobadas y no realizadas; a los recursos insuficientes frente a las demandas; a los déficits fiscales municipales; a la falta de participación popular; a disputas y tensiones entre partidos políticos y al sistema político clientelar... Sin la introducción de nuevos modelos de gestión pública (funcionamiento con programas y objetivos, evaluación y responsabilización de las diferentes áreas municipales, transparencia, nue- 
vos métodos presupuestarios y de contabilidad, capacidad de acción transversal de las varias administraciones, etc.), la eficiencia del proceso participativo se verá reducida.

Pero no todo son dificultades, la implementación de experiencias de participación presupuestaria también reportan beneficios. En primer lugar, es importante mencionar la transparencia ${ }^{31}$ ya que, por un lado, la administración municipal debe poner en conocimiento de la población su situación financiera antes de iniciar el proceso de discusión de las prioridades y, por otro lado, permite a la población involucrarse en temas que tradicionalmente fueron el campo de acción de los «expertos» debido a la especificidad de la temática y el nivel de especialización con que se debía contar para tratar estos temas. Además la transparencia del proceso presupuestario mejora la calidad del proceso democrático al facilitar la «rendición de cuentas» del gobierno (accountability) y la evaluación de su actuación por parte de los electores, quienes pueden conocer las prioridades de gasto público, su eficiencia, $y$, por consiguiente, votar con base en un análisis retrospectivo del grado de cumplimiento de las promesas electorales.

En segundo lugar, la práctica de la negociación y la definición de prioridades a través de un proceso público y abierto a la población en general. Esta situación provoca un aprendizaje en la población y permite también el surgimiento de nuevas élites políticas.

En tercer lugar, la credibilidad en las políticas públicas favorecida por la interacción entre la administración municipal, las asociaciones de vecinos y la población produce beneficios materiales concretos para las ciudades e indica no sólo una alteración de las prácticas políticas tradicionales sino una forma diferente de relación entre el estado y la sociedad civil y la corresponsabilidad de los ciudadanos en la gestión de la ciudad.

La participación de los ciudadanos en el presupuesto público puede llegar a convertirse en 'el lugar' donde intentar reconstruir -con el tiempo y de manera colectiva- el concepto de 'bienes comunes', transformando las tensiones sociales en un 'proyecto compartido' enmarcado en espacios autogestionados por la sociedad civil y marcados por un diálogo interactivo con las instituciones correspondientes. Entre sus objetivos (de manera independiente o mediante asociaciones) cabe encontrar el desarrollo ético de las instituciones, el aumento del sentido cívico de los habitantes

\footnotetext{
31 FUENTES QuinTANA afirma que «la transparencia es al sector público lo que el mercado al sector privado».
} 
y de su capacidad para interpretar adecuadamente la complejidad del territorio, así como el reequilibrio de las distorsiones generadas por la sociedad de mercado, la ampliación del 'derecho a la ciudad' para todos aquellos que viven en ella y la difusión de formas de 'solidaridad negociada' que permitan la redistribución justa de los recursos públicos a favor de las categorías con mayores desventajas culturales, sociales y económicas.

Pero si eso no ocurre y como ha sucedido con otros temas desaparece de las agendas políticas siempre quedara el hecho de haber recuperado ideas olvidadas como: la transparencia, la rendición de cuentas, el consenso, la equidad, la política...

En cuanto a las experiencias estudiadas señalar que a pesar de tener pocos rasgos comunes, valen más por su diversidad, por su flexibilidad y por su adaptación rápida a los contextos locales que por su uniformidad. Estas experiencias no existen en forma aislada. Forman parte antes que nada, de una cultura popular de participación y de relaciones entre el gobierno local y la sociedad. Suponen como precondición, una ciudadanía movilizada.

\section{BIBLIOGRAFÍA}

ABERs, R. 1998: «Learning Democratic Practice: Distributing Government Resources Through Popular Participation in Porto Alegre, Brazil». En Mike Douglass and John Friedmann (Editores), Cities for Citizens. Chichester: John Wiley \& Sons.

Allegretti, G y HerZBerg, C. 2004: «El «retorno de las carabelas». Los presupuestos participativos de América Latina en el contexto europeo» en TNI Briefing Series, n. ${ }^{\circ} 2004 / 5$.

ARENILLA, M. 2004: La reforma administrativa desde el ciudadano. INAP. Madrid.

AVILÉs, J. 2004: «Técnicas de organización de los Presupuestos Participativos» en Temas para el Debate, Madrid, Sistema, no 113; 45-49.

Avritze, L. 1999: «Public Deliberation at the Local Level: Participatory Budgeting in Brazil» http://www.ssc.wisc.edu/ wright/avritzer.pdf

Bacque, M.H., Rey, H., Sintomer, Y. (coord.) 2004: Gestion de proximité et démocratie participative: les nouveaux paradigmes de l'action publique?, La Découverte, París. 
BAIERLE, S. 2003: «La experiencia brasileña en Presupuesto Participativo» en Documento Base del Seminario de Lanzamiento de la Red Urbal $n^{o}$ 9, Porto Alegre.

Barceló, S. y Pimentel, Z. 2002: Radicalizar la democracia. Porto Alegre: un modelo de municipio participativo. Ed. Catarata. Madrid.

Blanco, I. 2003: «Presupuestos Participativos en Rubí: innovación, límites y retos de una experiencia pionera» en Jornadas sobre participación ciudadana «Tu decides», Ayuntamiento de Jerez de la Frontera, 29-31 de octubre.

CABANNES, Y. 2003: «Introducción y Metodología» en Documento Base del Seminario de Lanzamiento de la Red Urb-al $n^{\circ}$ 9, Porto Alegre.

- 2003a: «Presupuestos Participativos en ciudades europeas y latinoamericanas: lecciones aprendidas de las experiencias» en Documento Base del Seminario de Lanzamiento de la Red Urb-al $n^{\circ}$ 9, Porto Alegre.

Fedozzi, L. 1997: Orçamento Participativo - Reflexões sobre a Experiência de Porto Alegre. Porto Alegre.Editorial Tomo.

GENRO T. 1997: «El presupuesto participativo y la democracia». http:// www.arrakis.es/ ldrain/demopunk/sp/direct/porto/porto1.html

- 2000: Reinventar el futuro. Democracia y socialismo en la era de la globalización. Ed. Del Serbal. Barcelona.

Gret, M., Sintomer, Y. 2003: Porto Alegre. La esperanza de otra democracia, Debate, Barcelona.

MarquetTi, A. 2000: Participatory budgeting in Porto Alegre. Indicator $S A$, Johannesburg, v.17, n.4, p.71-78.

- 2003: «Participação e Redistribuição: o Orçamento Participativo em Porto Alegre». Inovação Democrática: O Orçamento participativo no Brasil. Sao Paulo. Cortes Editora.

NAVARRO, Z. 2000: «Inventando o futuro das cidades: pequena história do Orçamento Participativo em Porto Alegre», en Rattner, Henrique (org.) Brasil no limiar do Século XXI. São Paulo, Edusp, p. 211-230.

- 2002: «O «Orçamento Participativo» de Porto Alegre (1989-2002): um conciso comentário crítico» Proyecto Democracia Participativa. 
Pérez Castell, M. 2004: «La experiencia de los Presupuestos Participativos en Albacete» en Temas para el Debate, Madrid, Sistema, $\mathrm{n}^{\circ}$ 113, 54-57.

PinedA, C. y SANZ, A. 1999: «Presupuesto y participación: la experiencia de Porto Alegre y Kerala», Ponencia presentada en el IV Congreso de Ciencia Política y de la Administración, Granada, 30 de septiembre-2 de octubre.

PINEDA, C. 2002: «Mejora de la gobernabilidad en el nivel de Gobierno local: participación de más actores en el proceso presupuestario», Ponencia presentada en el VI Congreso del CLAD, Lisboa, 8-11 octubre.

- 2002a: «Gobiernos locales: participación ciudadana en el proceso presupuestario» en Revista de Gestión y Análisis de Políticas Públicas, Madrid, INAP, $n^{\circ}$ 21, 161-173.

- 2003: «La participación ciudadana en el proceso presupuestario dentro de ámbito local: consideraciones generales y la experiencia de Albacete», Ponencia presentada en el VI Congreso de Ciencia Política y de la Administración, Barcelona, septiembre.

- 2004: «Posibilidades y condicionamientos de los Presupuestos Participativos» en Temas para el Debate, Madrid, Sistema, $\mathrm{n}^{\mathrm{o}}$ 113, 41-45.

- 2004a: «Los Presupuestos Participativos desde una perspectiva comparada», Ponencia presentada en el VIII Congreso de Sociología, Alicante, 23-25 septiembre.

- 2004b: «Los Presupuestos Participativos en España: un balance provisional», Revista de Estudios Locales, n 78, 64-76.

PIRES, V. 2000: «Límites y potencialidades del presupuesto participativo». Revista Internacional de Presupuesto Público (ASIP). Número 42. http://www.asip.org.ar/es/revistas/.

- 2000a: «Participación de la sociedad en los procesos presupuestarios: la experiencia brasileña reciente». Ponencia presentada en el XXVII Seminario Internacional de Presupuesto Público. Madrid, noviembre.

Rodríguez Álvarez, J.M. 2004: La Ley de Medidas para la Modernización del Gobierno local. Ed. Bayer. Barcelona.

Rodríguez Prieto, R. 2005: Ciudadanos soberanos. Participación y democracia directa. Ed. Almuzara. Madrid. 
SANTOS, B. 1998: «Participatory budgeting in Porto Alegre: towards a redistributive justice». Politics and Society, v. 26, n. 4, 416-510.

- 2002: «Orçamento Participativo em Porto Alegre: para uma democracia redistributiva», en Santos, Boaventura de Sousa (org). Democratizar a democracia. Os caminhos da democracia participativa. Rio de Janeiro, Civilização Brasileira, p. 455-560.

SinTOMER, Y. 2004: Los presupuestos participativos en Europa: retos y desafíos. Comunicación presentada en el IX Congreso Internacional del CLAD sobre la Reforma del Estado y de la Administración Públi$c a$, Madrid, España, 2-5 noviembre.

Utzig, J. E. 1999: «Orçamento Participativo e Performance Governamental». paper escrito para el programa SPURS (Special Program for Urban and Regional Studies) en el MIT (Massachusetts Institute of Technology), Cambridge, Estados Unidos, en el primer semestre de 1999.

- 2000: El presupuesto participativo en Porto Alegre: análisis basado en el principio de legitimidad democrática y el criterio de desempeño gubernamental. Documento publicado por el Banco Mundial.

Villasante, T. 1994: Los desbordes de técnicos y vecinos en los movimientos ciudadanos actuales, El Viejo Topo, Barcelona.

WAMPLER,B.2002: «Orçamento participativo: uma explicação para as amplas variações nos resultados». Inovação Democrática: O Orçamento participativo no Brasil, Sao Paulo. Cortes Editora. 


\section{Anexo I}

Criterios para la priorización de propuestas del foro participativo de Albacete

\begin{tabular}{|c|c|c|c|}
\hline CONCEPTO & NOTA & ACTUACIÓN & PUNTOS \\
\hline \multirow{4}{*}{$\begin{array}{c}\text { Participación } \\
\text { Ciudadana }\end{array}$} & \multirow{4}{*}{2} & Vivienda & \\
\hline & & Administración (cercanía y calidad) & \\
\hline & & Seguridad ciudadana & \\
\hline & & Medio ambiente & \\
\hline \multirow{4}{*}{ Acción Social } & \multirow{4}{*}{3} & Educación y cooperación & \\
\hline & & Salud y consumo & \\
\hline & & Empleo & \\
\hline & & Cultura, ocio y deporte & \\
\hline \multirow{4}{*}{ Movilidad } & \multirow{4}{*}{4} & Transporte y comunicaciones & \\
\hline & & Accesibilidad & \\
\hline & & Calzada & \\
\hline & & Aceras y espacio peatonales & \\
\hline \multirow{4}{*}{$\begin{array}{c}\text { Equipamiento } \\
\text { Urbano }\end{array}$} & \multirow{4}{*}{1} & Equipamientos e infraestructuras municipales & \\
\hline & & Alumbrado y suministro & \\
\hline & & Reciclado y abastecimiento & \\
\hline & & Mobiliario & \\
\hline
\end{tabular}

